\title{
Creepex as a parameter of seismo-geodynamic studies based on geo-information systems
}

\author{
Anna V. Mikheeva ${ }^{1}$, Igor I. Kalinnikov ${ }^{2}$ \\ ${ }^{1}$ Institute of Computational Mathematics and Mathematical Geophysics SB RAS, Novosibirsk, Russia \\ ${ }^{2}$ Institute of Physics of the Earth of O.Yu. Schmidt RAS, Moscow, Russia
}

\begin{abstract}
The GIS-EEDB (the Expert Earthquake Database) and GIS-ENDDB (the Earth's Natural Disasters DataBase) geoinformation systems implement methods for spatial-temporal analysis of both classical and new characteristics of the seismogeodynamic process. One of the new characteristics is a normalized creepex parameter that can be used in the statistical approach to studying of seismicity to assess a relative contribution of "soft" (creep) and "hard" (explosion) movements to the overall process of focal radiation. In this paper, the following modifications of the creepex parameter are proposed and compared: 1) $C r_{0}-C r_{0 \_}$world and $C r_{N}-C r_{N \_w o r l d}$ as the result of reducing the parameters $C r_{0}$ and $C r_{N}$ of each event to the world average values of the creepex, obtained from the polynomial dependence of $C r_{i_{-} \text {world }}(i=0, N)$ on $M_{S_{-} \text {world }}$, where $C r_{i_{-} w o r l d}$ are calculated based on the world average estimates of $M_{S_{-} \text {world }}$ and $m_{b_{-} \text {world }}$ at uniform intervals of the seismic moment (NEIC catalog), 2) $C_{r_{-} b j i}$ as the result of reducing $C r_{N}$ of each event to $C r_{N_{-} b j i}$, i.e. to the average for the studied in this work BJI catalog creepex values obtained from the polygonal trend $C r_{N}\left(M_{S}\right)$ of all the catalog events. The advantage of using the creepex parameter, reduced to the trend of averaging the magnitudes of the catalog in question, is revealed, since the smallest linear dependence of the creepex on the magnitude is achieved (in comparison with the classical and normalized creepex) and the symmetry in the maximum amplitude of the deviation of the parameter relative to zero is preserved. Examples of the application of the compared parameters in specific seismic-geodynamic studies of aftershock processes of the Molucca and Simushir earthquakes also demonstrate the advantage of the parameter $C_{r_{-} b j i}$ in the observed correspondence of the variations of the latter to the known physical processes in the focal zone of the Kashmir event, studied by the seismic-geodynamic methods of the GIS-EEDB geographical information system.
\end{abstract}

\section{Keywords}

Catalogs and databases of earthquakes, seismic and geodynamic processes, tectonic environments.

\section{Introduction}

At the present time, several modifications and prototypes of GIS-EEDB (the Expert Earthquake Database) and GIS-ENDDB (the Earth's Natural Disasters DataBase - a database of disastrous events: earthquakes and impact structures) [1] are functioning in various scientific and educational organizations. Its implement methods of statistical spatial-temporal analysis of the characteristics of the seismogeodynamic process: both the classical (parameters of the repeatability graph, the total number of earthquakes, the total seismic energy, methods of transformation of geophysical fields, etc.) and the new (the relative total seismic energy and

SDM-2021: All-Russian conference, August 24-27, 2021, Novosibirsk, Russia

$\bigoplus$ anna@omzg.sscc.ru (A.V. Mikheeva); i-kalinn@yandex.ru (I. I. Kalinnikov)

(c) (1) 12021 Copyright for this paper by its authors. Use permitted under Creative Commons License Attribution 4.0 International (CC BY 4.0).

CEUR Workshop Proceedings (CEUR-WS.org) 
its gradients; maximum earthquake depths, the total slip direction in seismic foci, methods of grouping events into seismolineaments, swarms, clusters; methods of correlation analysis, etc.). Recently, a method for calculating the normalized creepex was implemented in the GIS system, which complements the complex seismogeodynamic analysis with maps and graphs of changes in this parameter in order to identify the relationship of seismic events with the tectonic environment (by the distribution of its anomalies in space), as well as the regularities of the seismogenesis processes before and after a strong event (by a change in the parameter in time).

\section{The concept of creepex, its applicability and modifications}

A formally constructed parameter of creepex (creep \& explosion) is determined by the ratio of magnitudes $M_{S}$ and $m_{b}$ :

- by the difference $C r_{0}=M_{S}-m_{b}$ [2] (other types of magnitudes for volume waves are also allowed [3]);

- by the residual of these magnitudes in the orthogonal regression $C r=M_{S}-k \cdot m_{b}-l[4]$.

From a mathematical point of view, a creepex is a difference of quantities of the same dimension, but with a different physical content. In fact, it is a normalized logarithm of the ratio of different estimates of the earthquake energy. The energy parameters of MS and mb are determined by different types (surface and volume) waves at different frequencies (a low-frequency range for MS: usually with a period of $20 \mathrm{~s}$; and a high-frequency range for mb: usually with a period of $1 \mathrm{~s}$ ). The ratio of MS and $\mathrm{mb}$ indicates the predominance of low or high frequencies in a spectrum, indicating macroseismic processes with the creep or the explosion effect, respectively. This allows the parameter to be used, for example, for reliable explosions identification on the background of seismic events of natural and anthropogenic origin. The spectral characteristics of the processes of fast and slow disruptions of the geophysical environment continuity act as an information carrier in the identification criteria. The extremely fast destruction by explosion is accompanied by the emission of high-frequency seismic vibrations, the extremely slow one is by a plastic flow, i.e. a creep, which in the extreme case is not accompanied by the highfrequency seismic emission (episodic tremor and sliding). But the information content of the creepex is much broader, since $M_{S}$ and $m_{b}$ are evaluated by seismologists based on the spectral characteristics of the wave forms associated with an earthquake, each reflecting the specific features not only of an earthquake itself, but also the conditions of its origin and propagation in the "muddy" (according to [5]) geophysical environment.

Thus, $\mathrm{Cr}_{0}$ and $\mathrm{Cr}$ are those simple parameters of the spectral characteristics of the earthquakes that can be used in the statistical approach to studying of seismicity to assess a relative contribution of "soft" (creep) and "hard" (explosion) movements to the overall process of focal radiation. Thus, the dominance of increased values of the creepex in the region under study, reflecting the predominance of low frequencies in the earthquake spectrum, is a sign of tectonic "softness" [3]. Indeed, in [6, 7], it is confirmed that in transform faults with a shift deformation or spreading zones, the tectonic movement in which generally exhibits the property of "aseismic creep", seismic sources have a much higher value of the creepex than in subduction zones 
characterized by a "cold" brittle fracture. On the other hand, the reason for the local differences in the creepex distributions "be sought for in different conditions for generating seismic waves in specific foci - the type of strength or mechanism of the focus" [2,3]. This means that local $\mathrm{Cr}$ deviations are "a measure of the specificity of the focal zone", and spatial-temporal changes in the parameter will be "due to the conditions variations in the focal zone on the radiation of compression and shift waves" [7]. However, a "balanced" approach is also necessary here, since the accuracy of the $\mathrm{Cr}$ estimate for an individual event, determined by the error in calculating $M_{S}$ and $m_{b}$, can be "both objective and subjective" [7, 8].

There are many factors leading to the dependence of the radiation efficiency of a seismic source on the scope of events, for example, the effect of absorption and scattering of high frequencies during the oscillations propagation [9]. In addition, there are some methodological problems in estimating the seismic energy of earthquakes, for example, its systematic overestimation in an elastic medium in comparison with a viscoplastic one [10], etc. Such factors can be the reason for the long-discovered nonlinearity of relationships between magnitudes of different types [11]. Thus, studying earthquake catalogs created by many agencies has shown that the difference between the magnitudes $M_{S}$ and $m_{b}$ depends on the earthquake magnitude $M_{S}$ according to the linearly growing law $[3,8]$. This dependence complicates the use of the creepex parameter in statistical studies, making it difficult to adequately interpret its anomalies without a special consideration of the magnitude of each event in a sample.

To solve this problem, a modified (normalized to the arithmetic mean of $M_{S}$ and mb of each event) estimate of the creepex parameter $C r_{N}=4\left(M_{S}-m_{b}\right) /\left(M_{S}+m_{b}\right)$ was proposed in [8] which somewhat reduced its systematic dependence on the earthquakes magnitude $M_{S}$ (especially pronounced for large events), but did not completely eliminate this dependence, transforming it from linear to logarithmic (Figure 1).

In this paper, the following modifications of the creepex parameter are proposed and compared.

1. $C r_{0}-C r_{0 \_w o r l d}$ and $C r_{N}-C r_{N_{-} w o r l d}$ is the result of reducing the parameters $C r_{0}$ and $C r_{N}$ of each event to the world average values of the creepex, obtained from the polynomial dependence of $C r_{i \_ \text {world }}(i=0, N)$ on $M_{S_{-} \text {world }}\left(\right.$ Figure 2, a), where $C r_{i \_w o r l d}$

a

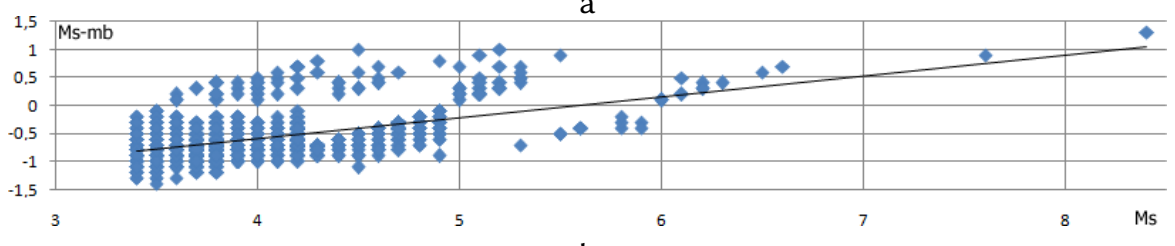

b

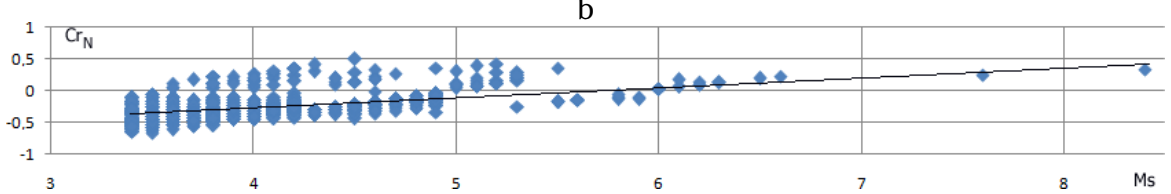

Figure 1: The dependence on the magnitude $M_{S}$ of the creepex-parameters $C r_{0}$ (a) and $C r_{N}$ (b) by an example of the Tohoku earthquake vicinity (1458 paired determinations: $11.03 .2010-11.03 .2012,30-50^{\circ} \mathrm{N}$; $\left.140-160^{\circ} \mathrm{E}\right)$. 
a

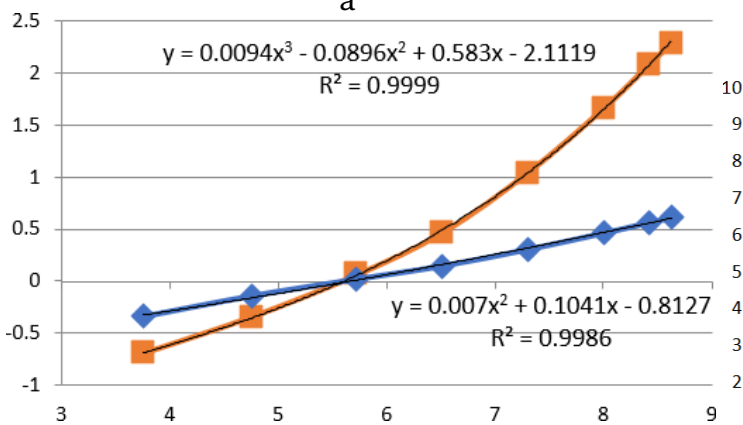

b

$\mathrm{MW}(\mathrm{Ms})$

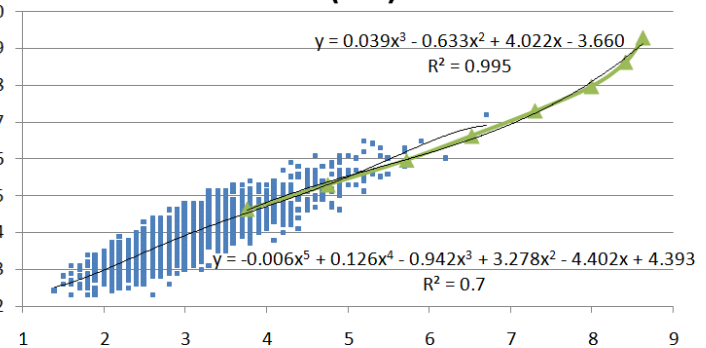

Figure 2: The polynomial dependences: a - on the average world magnitude $M_{S_{-} \text {world }}$ of the creepexparameters: $C r_{0 \_w o r l d}=M_{S \_w o r l d}-m_{b_{-} w o r l d}($ red $)$ and $C r_{N \_w o r l d}$ (blue); b - of the world average magnitude $M_{W}$ on the $M_{S_{-} \text {world }}$ (green) and of the magnitude $M_{W}$ (calculated from $\left.M_{L}\right)$ on $M_{S_{-}}\left(M_{S}\right.$ corrected for the trend level) for all the events in the BJI catalog (blue).

are calculated based on the world average estimates of $M_{S_{-} w o r l d}$ and $m_{b \_w o r l d}$ [11] at uniform intervals of the seismic moment (NEIC catalog).

2. $C r_{b} j i$ - the result of reducing $C r_{N}$ of each event to $C r_{N_{-} b j i}$, i.e. to the average (for the studied in this work BJI catalog [12]) creepex values obtained from the polygonal trend $C r_{N}\left(M_{S}\right)$ (black line in Figure 2, b) of all the catalog events.

\section{Comparative analysis of creepex modifications, examples of their application}

Comparison of the proposed parameters can be carried out both from the view point of solving the problem of the creepex dependence on magnitude, and based on the correspondence of the creepex variations to the known physical processes in the foci of specific events, investigated by different methods of a geographic information system.

The dependence $M_{S}$ of different creepex versions is shown in Figure 3 using an example of the aftershock swarm of the January 21, 2007 earthquake $\left(M_{S}=7.5\right)$ in Molucca according to the BJI catalog. The reduced to the average trend of the BJI catalog creepex value $C r_{N}-C r_{N \_b j i}$ demonstrates a less pronounced dependence on magnitude than the parameter $C r_{N}$, while maintaining the character of monotonic growth with an increase in the magnitude of the event (Figure 3, a).

On the contrary, the creepexes, reduced to the global data (calculated by NEIC [11]): $C r_{N}-$ $C r_{N \_w o r l d}$ and $C r_{0}-C r_{0 \_w o r l d}$ demonstrate (Figure 3, a) the suppression of the monotonic growth at large magnitudes, resulting in distortion of information about the creepex for large events. This may be due to a systematic difference in estimates $M_{S}$ presented by different catalogs (here, by BJI and NEIC ones). It is possible, of course, to find the correspondence of these estimates by the coincidence of the trends in their relationship with a more objective by defined magnitude $M_{W}$ (Figure 2, b). We have estimated the latter according to the magnitude $M_{L}$ presented in the BJI catalog. It was found that, in contrast to the dependence $M_{W}\left(M_{L}\right)$ : $M_{W}=M_{L}-0.4$ according to Abubakirov et al. [13], for BJI a good agreement of the trends 
a

b

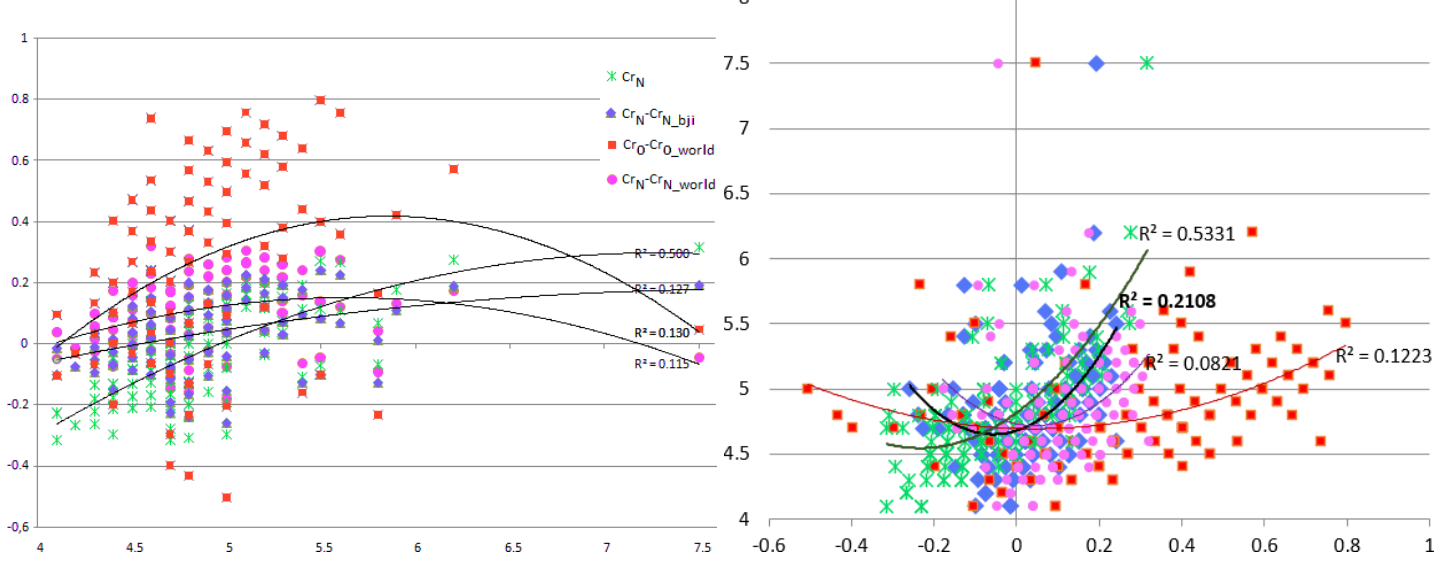

Figure 3: Dependence on the magnitude MS of various creepex parameters: $C r_{N}, C r_{N}-C r_{N \_w o r l d}$, $C r_{0}-C r_{0 \_ \text {world }}$ and $C r_{N}-C r_{N_{-} b j i}$ on an example of the aftershock swarm of the earthquake of 21.01.2007, $M_{S}=7.5$ in Molucca.

in the area of their intersection is given by the formula $M_{W}=M_{L}+0.5$. If we accept $M_{W}=M_{L}-0.4$ [13], then it is necessary to decrease the estimate $M_{S}$ in the BJI catalog by 1.2: $M_{S_{-}}=M S-1.2$ (Figure 2, b). For $m_{b}$ in this case, a correction -0.4 is required. The introduction of such significant corrections brings about a large shift of the creepex $C r_{N}$ value (and, largely, of the value $C r_{0}$ ) into the area of negative values. Conversely, reducing the values $C r_{0}$ and $C r_{N}$ to average the $C r_{\text {world }}$ and $C r_{N \_ \text {world }}$ world creepexes, obtained from relatively high magnitudes, leads to a shift of the obtained estimates into the positive area (Figure 3, b; yellow and gray lines in Figure 4, a). Apparently, the events distribution shifted in one or another direction does not correspond to the equal importance of the contributions (to seismicity, in general) of two physical processes: a brittle fracture and a viscous slip. Thus, from the viewpoint of the expediency of using the creepex, it does not allow to visually classify events according to relevant two groups using the obtained parameters.

So, the idea of reducing the creepex to the average value over the world catalogs (NEIC) for the events of the BJI catalog cannot be accepted because, although it removes the dependence of the creepex on $M_{S}$, leads to a systematic shift of its normalized estimate to the area of negative or positive values. Now consider the graph in Figure 3, b showing the advisability of reducing the creepex to the trend of averaging the itself magnitudes of the catalog used, in this case: $C r_{b j i}=C r_{N}-C r_{N_{-} b j i}$. The distribution of this parameter (its trend is highlighted by a bold line) has no displacements about the zero axis.

Examples of the application of the compared parameters in specific seismic geodynamic studies on various aftershock processes (Figure 4) also demonstrate the advantage of the parameter $C r_{b j i}$. The first, strongest shock (marked with vertical lines in Figure 4) on the graphs of $C r_{N}$ and $C r_{b j i}$ (red and blue colors) is marked by a regular significant increase in the creepex value (which shows a large contribution of quasi-plastic flow to the mechanism of main shocks, and sometimes of several subsequent shocks), after which a gradual (over several hours) decline occurs, characterizing the beginning of the medium conversion to brittle fracture 


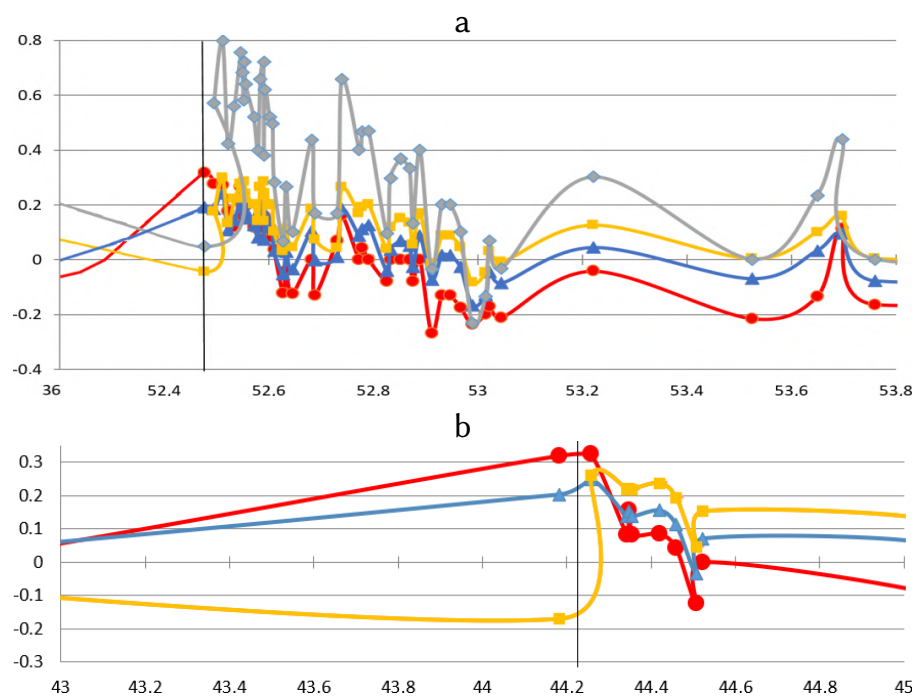

Figure 4: Changes in various creepex parameters in time. a - a 1.5-day fragment of the aftershock swarm of the earthquake of January 21, 2007, $M_{S}=7.5$ in the Moluccan Sea, Indonesia (at the junction of the Eurasian plate and the Papua microplate): $C r_{N}$ (red color), $C r_{N}-C r_{N_{-}} w o r l d$ (yellow), $C r_{0}-C r_{0 \_w o r l d}$ (gray) and $C r_{b j i}$ (blue); b - a 0.5-day fragment of the aftershock swarm of the Simushir earthquake of January 13, 2007, $M_{S}=8.1$ (at the junction of the Okhotsk and the Pacific plates): $C r_{N}$ (red), $C r_{N}-C r_{N \_w o r l d}$ (yellow) and $C r_{b j i}$ (blue).

(developed the next day). A similar mechanism was established as a result of a more detailed geodynamic study of the Kashmir earthquake $\left(08.10 .2005, M_{S}=7.6\right)$ carried out by means of the GIS-ENDDB system (Figure 5).

The temporal distribution of aftershocks of the Kashmir earthquake (Figure 5, a) clearly demonstrates the relaxation process of the source in the first hours after the main shock by partial, periodic chains of events with a decreasing value of the creepex $C r_{N}$ within a chain. According to a change in the value $C r_{N}$, the chains demonstrate the transition from quasiplastic flow to brittle fracture, according to the general interpretation from [6]. Let us note that a number of studies $[14,15]$ mention periodic activations of the after-shock process of the Kashmir earthquake and the impossibility of explaining its without introducing special corrections into the seismodynamic model (in particular, the "slip variability" correction, "repeated slip", etc.) or the assumption of the existence of a highly fluid layer in the lower crust.

The connection of the initial events of each chain with quasi-plastic processes at depth is also proved by their depths: the temporal distribution of the ISC catalog events near the focal zone shows that at the beginning of each chain there is a deeper event than the subsequent ones in the chain, and the main shock of the Kashmir earthquake, although confined to the depth of the brittle layer ( $H=8.2 \mathrm{~km}$ according to ISC data), is preceded by two foreshocks ( 3 hours before the event), which have a greater depth: 15 and $14 \mathrm{~km}$ (Figure 5, b). Let us also note that according to the data of the CMT global catalog [16], the mechanisms of the overwhelming number of events in the Kashmir earthquake source are an upthrust (Figure 5, c), determined by the tectonic setting of collisional compression, covering a large range of depths.

The graph of $C r_{\text {ISC }}$ changes over time is more smoothed (the red line in Figure 5 , d) than 


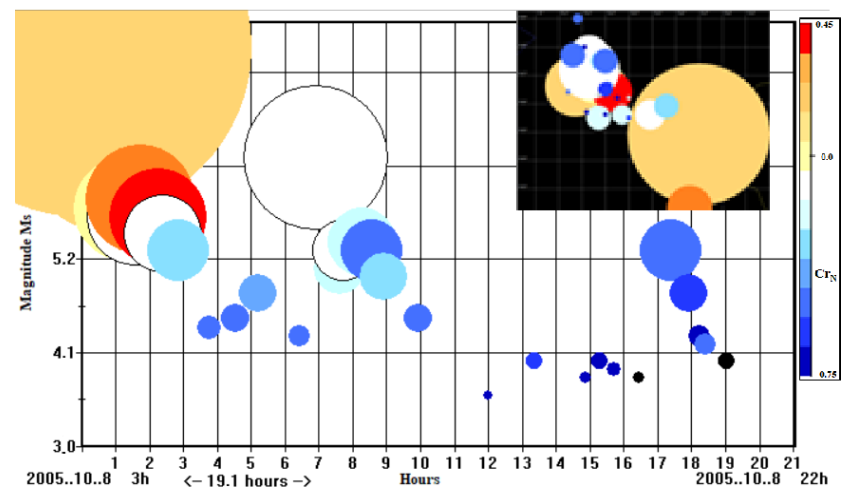

C b

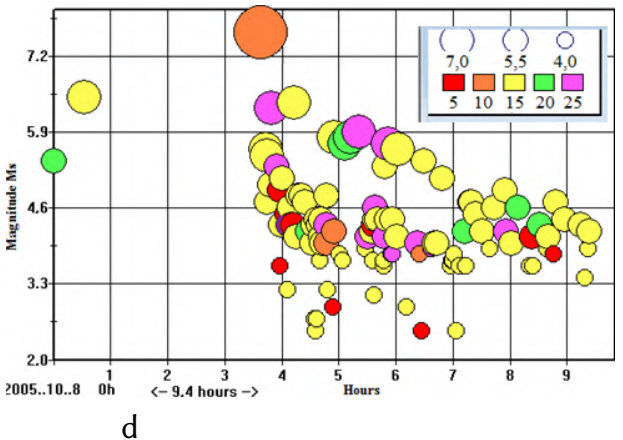

d

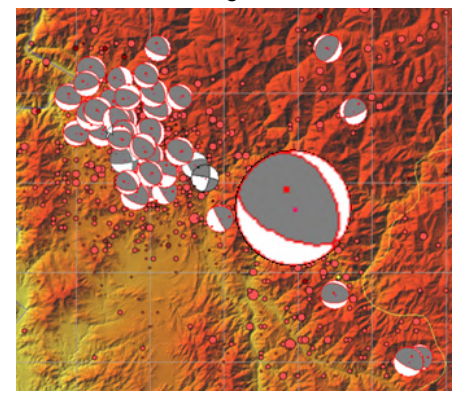

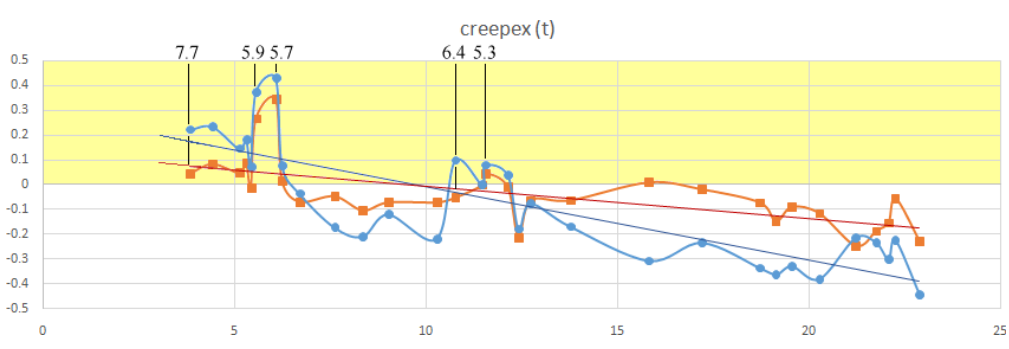

Figure 5: Development in time (the first day) of the seismogeodynamic process in the focal zone of the Kashmir shock (lcreepex catalog containing the paired ISC definitions of $M_{S}$ and $m_{b}$ ): a - the events colored by the creepex value $C r_{N}$, where the size of the circles is set by the focal zone size estimated by $M_{S}$ [17]; in the inset picture: distribution of these events in the map (all the pictures were obtained by the GIS-ENDDB analysis system); b - all events of the ISC catalog in the study zone colored by depth (color and radius are determined by the legend: $H$ in $\mathrm{km}$ by color, $M_{S}$ by size); c - the mechanisms of the Kashmir earthquakes for 2004-2016: $\mathrm{d}-$ a change in the values of $C r_{N}$ (blue) and $C r_{\text {ISC }}$ (red) in the sample of Figure 5, a.

the $C r_{N}$ graph (the blue line), since stronger events at the beginning of each chain are not manifested here by the creepex increase (because there is no proportional dependence of the creepex on the magnitude). Apparently, the $C r_{\text {ISC }}$ graph more objectively reflects the physical processes in the focus: the main shock (which is marked by the first point of each chart) does not show up on the $C r_{\text {ISC }}$ chart as either a positive or a negative maximum. But 1.5 hours after it, the sharp maximum marks the greatest contribution of the viscous slip to the mechanism of a pair of synchronous aftershocks $\left(M_{S}=5.9\right.$ and $\left.M_{S}=5.7\right)$ located along the perimeter of the focal zone of the main shock (red and orange circles in the inset picture of Figure 5, a), which can characterize a sharp change in the environment state caused by the destructive upthrust of the main shock. Another 5 hours later, an aftershock with $M_{S}=6.4$ (the beginning of the second chain of aftershocks in Figure 5, a) occurs just to the northwest of the main shock, which also does not show up on the $C r_{\text {ISC }}$ chart as a positive maximum, but an hour after which there is a jump of $C r_{\text {ISC }}$ to the area of positive values (Figure 5, d). It is possible that both of these maxima show a pattern of occurrence of aftershocks with an increased creepex value after the largest aftershocks. 


\section{Conclusion}

The creepex is an informative parameter reflecting the properties of the geophysical environment, the dynamic processes in which are manifested in the spectral features of waveforms generated by earthquakes. Obtaining a quantitative measure of information extracted from the creepex requires taking into account the peculiarities of the used information base: catalog of earthquakes. This paper shows the advantage of using the creepex parameter, reduced to the trend of averaging the magnitudes of the catalog in question, since a smaller linear dependence of the creepex on the magnitude is achieved (in comparison with classical creepex) and a symmetry is preserved in the maximum amplitudes of the parameter deviation relative to zero. The choice of the most informative modification of the creepex parameter and its implementation into the expert information and computing system GIS-ENDDB will provide the possibility: to calculate and visualize the distribution of the creepex parameter in space and time; to trace its retrospective dynamics in various regions, including that in the zones of preparation and development of strong earthquakes; to identify possible predictive patterns in the resulting anomalies. For example, the researching Kashmir earthquake (08.10.2005, $M_{S}=7.6$ ) by means of the GIS-ENDDB system demonstrates in terms of the creepex value change the patterns of medium transition from quasi-plastic flow to brittle destruction after a major shock.

\section{Acknowledgments}

This work was carried out under state contract with ICM\&MG SB RAS 0251-2021-0004.

\section{References}

[1] Mikheeva A.V. Geostructural elements identified by mathematical algorithms and digital models of the GIS-ENDDB geographic information and computing system. Novosibirsk, 2016. 300 p. (In Russ.).

[2] Prozorov A.G., Hudson D. Dependence between MLH and MPV on regional conditions and local interconnections // Magnitude and Energy Classification of Earthquakes. Vol. 2. Moscow: IFZ AN SSSR, 1974. P. 208-216. (In Russ.).

[3] Neverova N.P. Creepex - characteristics of the earthquake source // Materials of the Fourth International Seismological School "Modern Methods of Processing and Interpretation of Seismological Data”. Obninsk, 2009. P. 127-129. (In Russ.)

[4] Kaverina A.N. Spectral-magnitude anomalies of seismic sources. PHd Thesis. Moscow, 1998. (In Russ.).

[5] Nikolaev A.V. Seismic turbidity of real media and the possibility of its study // Dokl. Academy of Sciences of the USSR. 1967. No. 177:5. P. 1072-1074. (In Russ.).

[6] Kaverina A.N., Lander A.V., Prozorov A.G. Global creepex distribution and its relation to earthquake-source geometry and tectonic origin // International Geophysical Journal. 1996. Vol. 125. Is. 1. P. 249-265.

[7] Boldyrev S.A., Levina V.I. Creepex of Kamchatka small-focus earthquakes // Physics of the Earth. 2008. No. 3. P. 40-57. (In Russ.) 
[8] Kalinnikov I.I., Mikheeva A.V. The Creepex-analysis of processes in large earthquakes focal zones by the GIS-ENDDB tools on the Tohoku example // Bulletin of the Novosibirsk Computing Center. Series: Math. Model. in Geophys. 2020. Vol. 22. P. 11-21.

[9] Besedina A.N., Kabychenko N.V., Kocharyan G.G. Features of seismic monitoring of weak dynamic events in a rock massif // FTPRPI. 2013. No. 5. P. 20-36. (In Russ.)

[10] Storcheus A.V. Notes on the methodology for calculating the seismic energy of explosions and earthquakes // Materials of the Conference Dedicated to the Volcanologist Day. March 27-29, 2008. Petropavlovsk-Kamchatsky: IVMS FEB RAS, 2008. P. 274-281. (In Russ.)

[11] Gusev A.A., Melnikova V.N. Relations between magnitudes are world average and for Kamchatka // Volcanology and Seismology. 1990. No. 6. P. 55-63. (In Russ.)

[12] Catalog of China Bureau of Earthquake Research BJI of Institute of Geophysics, Academy of Sciences of China. Available at: http://www.isc.ac.uk/cgi-bin/web-db-v4?request= COMPREHENSIVE\&out_format (accessed May 13, 2021).

[13] Abubakirov I.R., Gusev A.A., Guseva E.M., Pavlov V.M., Skorkina A.A. Mass determination of moment magnitudes $\mathrm{Mw}$ and establishing the connection between Mw and ML for moderate and weak Kamchatka earthquakes // Physics of the Earth. 2018. No. 1. P. 37-51.

[14] Javed F., Hainzl S., Aoudia A., Qaisar M. Modeling of Kashmir aftershock decay based on static Coulomb stress changes and laboratory-derived rate-and-state dependent friction law // Pure and Applied Geophysics. 2016. Vol. 173. Is. 5. P. 1559-1574.

[15] Wang K., Fialko Yu. Space geodetic observations and models of postseismic deformation due to the 2005 M 7.6 Kashmir (Pakistan) earthquake // J. Geophys. Res.: Solid Earth. 2014. Vol. 119. P. 7306-7318.

[16] Global CMT Catalog Search. Global CMT Web Page-Lamont-Doherty Earth Observatory (LDEO) of Columbia University, Columbia, SC, USA. Available at: http://www.globalcmt. org (accessed Mat 13, 2021).

[17] Riznichenko Yu.V. Dimensions of the crustal earthquake focus and seismic moment // Research on the Physics of Earthquakes. Moscow: Nauka, 1976. P. 9-27. 